\title{
EXPEDITIOUS METHODS OF URBAN SURVEY FOR SEISMIC VULNERABILITY ASSESSMENTS
}

\author{
G. Predari ${ }^{1}$, C. Bartolomei ${ }^{1}$, C. Morganti ${ }^{1}$, G. Mochi $^{2}$, R. Gulli ${ }^{1}$
}

\author{
${ }^{1}$ Department of Architecture, Alma Mater Studiorum - University of Bologna \\ (giorgia.predari, cristiana.bartolomei, caterina.morganti4, riccardo.gulli)@unibo.it \\ ${ }^{2}$ Department of Civil and Environmental Engineering - University of Perugia \\ giovanni.mochi@unipg.it
}

\section{Commission II}

KEY WORDS: Digital Heritage Documentation, Structure From Motion, Building aggregates, Seismic vulnerability, Historical centres, Imola

\begin{abstract}
:
The aim of the proposal is to illustrate how expeditious procedures of urban survey carried out through photomodeling can be the most suitable representation tool in combination with an expeditious procedure for assessing the seismic vulnerability of the historic building heritage in Italian city centres. For some years, in fact, the research group is developing a protocol for the rapid assessment of the seismic vulnerability of masonry aggregate buildings in Italian historic centres.

The protocol is based on the determination of synthetic indicators providing a preventive quantification of the possible earthquake damage. This evaluation procedure is oriented to prevent and reduce the current vulnerability, aiming at the conservation and preservation of the historic building heritage. The synthetic indicators are defined by identifying expeditious evaluation procedures based on the typical evolutionary processes suffered by each aggregate in its planimetric and height development, on the construction techniques and on the design concepts used in the local area; these aspects are directly correlated to failure modes.

The application of the entire process (starting from the rapid survey phases up to the final restitution of the seismic vulnerability assessment results) is illustrated for the historic centre of Imola. The ancient nucleus of this city constitutes an excellent example, as it is clearly representative of the Emilian historical architecture both for the processes of formation and transformation of the inhabited area, both for what concerns the constructive characterization deriving from the local building traditions
\end{abstract}

\section{INTRODUCTION}

The choice to focus the theme of architectural survey on masonry building aggregates to assess their seismic vulnerability derives from the recognition of how the measuring and documentation of the built heritage can provide very useful punctual analyses for the purposes of evaluation.

The activity of a geometric-architectural survey inevitably constitutes the morphological basis for all subsequent analyses and it must provide precise information on the actual state of the built heritage, and especially for building aggregates.

The survey must highlight certain aspects, as the identification of the structural types, the reading of the historical evolution of the building aggregate, any architectural relevance, the crack pattern, the mechanics of instability, the surfaces relating to the cadastral properties, the structural units and all the information necessary for a complete documentation and for a critical and objective reading on the real conditions of the aggregate.

As recognized by the "Guidelines for the assessment and reduction of seismic risk of cultural heritage": "The safeguarding of cultural heritage from the seismic risk in Italy is first of all a preventive issue, so far not implemented, except in singular cases (...) From this assumption, the need to acquire adequate tools for the conservation of cultural heritage in relation to the seismic action derives, and it is necessary to have analysis tools at different levels, which must be applicable at two different scales: the vulnerability assessment of the cultural heritage on a territorial scale, and the safety assessment and the intervention planning on the single building" (Ministry of Cultural Heritage and Activities, 2006).
The appropriate tools, cited by the guidelines, have been strongly affected by the transition from analogical to digital culture, continuing to mark a radical change in the field of architectural and urban surveying (Bianchini, Ippolito, Bartolomei, 2015).

Today, two-dimensional and three-dimensional digital objects require specific qualities in addition to a correct geometric representation.

The activity of associating useful contents to the representation of three-dimensional models, born from the photomodeling, has proved successful for the study of the seismic vulnerability at the scale of the urban fabric, which is a field regarding the urban investigation (Azhar, 2011).

"The urban survey is responsible for the extremely delicate role of identifying, highlighting, recognizing, relating, describing and finally representing, the set of values present in the investigated realities, in order to construct the set of formal and structural invariants present in the construction of the image of a project" (Coppo, 2010) so Dino Coppo summarized the peculiarities of urban survey.

Therefore, urban survey is intended as an open system of knowledge, where all the aspects can contribute to reconstruct the evolutionary history of the built environment of any city.

To illustrate this process, the city of Imola has been chosen, which is located about $30 \mathrm{~km}$ far from Bologna, on the Via Emilia where the Santerno Valley flows into the Po Valley.

In 2019, the historic centre of the city was involved in an integrated survey campaign which was divided into a wide range of operations that could be divided into two categories: the first refers to all the surveys aimed at knowing the geometry 
of the building aggregates, such as the direct and instrumental survey conducted with photomodeling; the second one includes all the operations aimed at highlighting the qualitative aspects of the built environment aimed at the evaluation of the seismic vulnerability (Barrile, Gelsomino, Bilotta, 2017).

The conducted study aims to propose both a high-quality methodology and a rapid analysis able to acquiring enough information to implement a high-level decision-making process to prevent earthquakes collapses in a reasonably short time. During the survey phases, this process allows to store a very big number of highly precise and always interrogable data, drastically reducing acquisition times and avoiding the need for continuous future on-site inspections with a considerable reduction in time and in costs.

The idea of this rapid approach derives from the succession of earthquakes that recently affected some parts of Italy.

\section{THE VULNERABILITY ASSESSMENT PROTOCOL}

Starting in the 1980s, the number of seismic events that cyclically affected the Italian territory increased the debate on the issue of seismic vulnerability of urban areas, aiming at the development of studies and analyses to investigate the theme of conservation and safety of the historical buildings. Urban environments are interpreted as the outcome of complex and articulated construction events that have defined their current appearance.

From this point of view, the current built heritage represents the testimony of centuries of transformations repeatedly implemented by its users for a progressive adaptation to their housing needs, according to mostly spontaneous growth models. Such stratifications in the construction phases give it a historical and testimonial value, which generally assumes cultural relevance. The fabric of historic centres must therefore be protected as an "extended cultural asset", since it is a "representation of the stratified culture of a community, a place of historical community and individual memories, identities and self-recognition of the population" (Consiglio Superiore dei Lavori Pubblici, 2012).

Regarding the problem of seismic vulnerability at the building scale, the incessant evolutionary process that has affected urban centres requires a specific and specifically dedicated approach. It must be able to consider the complexity of this constructed reality and be based on the awareness of the presence of artefacts that can be attributable to different constructive and structural types, even if mainly using masonry solutions.

The building aggregate is identified as an isolated and nonhomogeneous group of structurally interconnected buildings, which interact if stimulated by a dynamic action, and whose configuration is the result of an evolutionary process of expansions, aggregations, elevations and subsequent transformations over time.

This issue especially involves the Italian territory, which is characterized by historical fabrics mainly made up of compact and dense building aggregates, which represent a significant part of the national built heritage.

So the concept of seismic vulnerability referred to building aggregates has a different meaning when compared to the same words in the case of isolated buildings.

For the latter, this concept can be expressed in the capacity of the building to absorb the seismic acceleration without producing negative effects on its usability, on the protection of users or on the loss of the asset.

For historical building aggregates, which derive from multiple phases of transformation and which are characterized by construction solutions linked to local traditions, the most appropriate meaning of seismic vulnerability is the evaluation of their behaviour under an earthquake not in absolute terms, but relatively to urban heritage.

The observation of the damages caused by seismic events has repeatedly shown how the vulnerability of building aggregates, that is the propensity to be damaged with a consequent reduction in functionality, cannot be simply interpreted as the sum of the vulnerabilities of individual buildings. Indeed, there is such a correlation between mutually interconnected building units that they can be considered as a whole building. Therefore, these are heterogeneous structural systems, whose seismic vulnerability is difficult to evaluate and requires a unitary approach, since it is not possible to estimate the behaviour of an individual building extracted from the whole aggregate.

Moreover, in the study of the seismic vulnerability of building aggregates, one of the objectives is the need to provide a valid aid to the technicians who must express a judgment on the behaviour of individual structural units belonging to a building complex, to propose the best intervention solutions.

Often, they do not have the possibility to reach an effective knowledge because they do not know the construction and transformation phases of the building complex in its entirety (Cangi, 2012). In this case, the concept of vulnerability of a building aggregate includes the possibility of obtaining useful information to reach a greater awareness in building interventions.

A research (mainly focusing on the historical centres of the area affected by the 2012 Emilia and the 2016 Umbria-Marche earthquakes) was begun to define significant and effective levels of vulnerability and to validate hypotheses and proposals on the definition of a methodology able to identify the situations of greater fragility and the potential damage to parts of the buildings before the earthquake occurs.

The studies of Antonino Giuffrè, and in particular the case of Ortigia, are the starting point of this research (Giuffrè, 1993). From that moment on, the focus has been shifted from the single building to the building aggregate as the minimal unit of the historical urban fabric.

As Giuffrè had already pointed out, "the earthquake does not disintegrate houses, but it selects the structural parts and the weakest technological solutions, causing damage and collapse through mechanisms that can be defined in advance".

In fact, recent Italian earthquakes have highlighted how the primary seismic vulnerabilities of historical buildings can be found in the connection systems between the construction elements and in the stratifications and transformations experienced over time.

In these terms, the vulnerability of building aggregates can be identified as the propensity to damage that is generated due to the construction lacks the building (Carocci, 2005).

The recent earthquakes in Emilia (2012) and Umbria-Marche (2016) have allowed to test the validity of the protocol for the assessment of seismic vulnerability of building aggregates as a method for prevention and protection of urban centres. These events had shown how this method could profitably allow to identify, in an initial phase, the damage that could be caused by the seismic action.

The proposed protocol requires to define different phases of study, first on the historical fabric, then on the building aggregates themselves.

The seismic vulnerability assessment derives from the calculation of indicators, which are defined through the analysis of the typical evolutionary processes that have affected the aggregates and through the investigation of construction techniques used in the local area (Cremonini, 1994).

The final result of the evaluation is an overall index, which is basically the sum of partial indices, each multiplied by its own weight, which is a sort of quantification of the relevance of each 
indicator, initially determined on an experiential basis and subsequently refined on a statistical basis.

First of all, the method is based on the assumption that the seismic damage of the historical building derives from the loss of stability of individual components, which are seen as rigid blocks moving due to the ground acceleration. Then, from the insufficient shear strength of the walls (Mochi, Predari, 2016).

This second deficiency derives from issues related to the building materials, but the first derives essentially from the formation and modification processes of the building fabrics. Consequently, the comprehension of the transformation phases becomes the key-point for the behaviour prevision (Lagomarsino, Ugolini, 2005).

The identification of the transformation phases, starting from the first layout of the urban settlement, constitutes the initial point of this process, which leads to the current situation through the comprehension of the layout extensions, superelevations, demolitions and reconstructions.

This initial phase can only be hypothesized when there is no specific, historical or archaeological documentation, although some indirect sources may constitute an interesting reference base (Mochi, 2009).

The assessment of the seismic vulnerability of building aggregates follows the collection of data.

The vulnerability has been defined through indicators which are significant for their causal relationship with the damage: out-ofplane collapse of the façades and of the tympanums, cracks due to the rafters, hammering due to constructive irregularities (as the presence of buildings having a reinforced concrete structure inside the aggregate), weak shear strength due to insufficient width of the masonry walls (figure 1).

In addition to these, there is another essential indicator which is included in the global aggregate behaviour; this refers to the entire construction, not only to the façades.

This indicator is the wall disconnection index, which allows to understand the possible damage to the internal parts of the buildings composing the aggregate.

The wall disconnection index is also essential since it allows to define the portions of façades that can be subjected to out-ofplane collapse, and the width of the fronts to be considered effective for the shear mechanism.

The operational basis for the graphic representation of the method are drawings of plans and elevations of the current state, suitable for the transfer of all the necessary information regarding the constructive solutions and transformative processes.

Since this is a quick assessment procedure, whose application is useful for entire and extensive historical fabrics before carrying out a detailed quantitative analysis, in the same way the operational tools must derive from rapid procedures, and not from complete direct surveys of the urban fabric.

For the planimetric reconstruction of the buildings, the most recent plants belonging to the cadastral archives are used, while the quickest and precise choice for the survey of the facades consists in the use of photomodelling.

The specific purpose of these operations is to identify the invariant elements capable of characterizing the urban environment, which can become the basis for expeditious analyses able of giving reliable answers on seismic vulnerability.

The resulting product is a three-dimensional model that has a fundamental characteristic: measurability.

The photorealistic model, which will be detailed later, is in fact constructed from the point cloud and has a margin of error of about $3 \mathrm{~mm}$. Therefore, the model is no longer a simple representation in a documentation process, but becomes the essential pivot of the documentation itself, allowing reliable analysis.

From this assumption, we can understand how actual survey techniques and three-dimensional representation provide a critical tool able of analysing, in an autonomous way, morphological, metric, material and degradation aspects, fundamental for understanding the complex investigated phenomenon (Beolchini, Milano, Antonacci, 2005).

Moreover, the same techniques act as an effective tool of "interdisciplinary cooperation" with a high sharing gradient, able to integrate and make explicit, on the basis of the shared spatial positioning data, specialized readings relating to different knowledge, such as those necessary for the evaluation of the systemic vulnerability.

Unfortunately, the various disciplines that deal with the study of urban environments tend to manage the results of their own research independently, creating many difficulties in the global and linked interpretation.

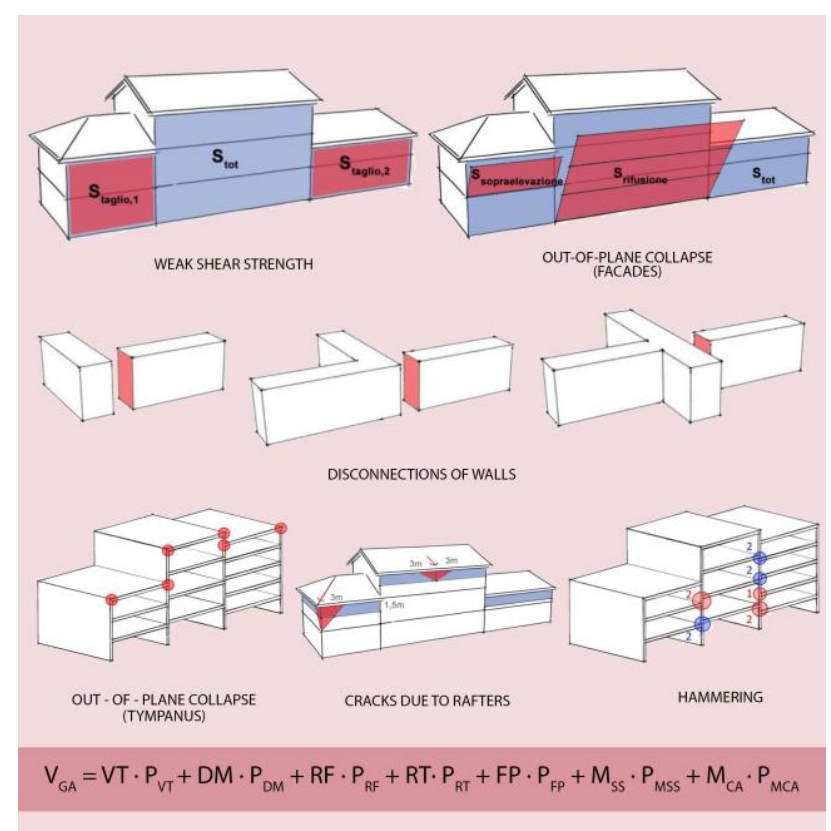

Figure 1. Indicators used for the seismic assessment

\section{THE APPLICATION ON THE HISTORICAL CENTRE OF IMOLA}

So far, the protocol had been applied to numerous Italian historical centres, in the North, in the Centre and in the South of the Country.

Through the collection of the described information, it was possible to define seismic damage scenarios, that were compared with the real manifestations of the 2012 and 2016 earthquakes.

For this case study, the historical centre of Imola was selected, as it is clearly representative of local construction techniques and it is located in a high seismic risk area.

The historical research initially allowed to identify the evolutionary phases of the city centre.

The city has grown in a close relationship with the surroundings: the settlement area is located between the plain and the base of the hills, an area favourable to human settlement due to the stability of the soil and the fertility of the agricultural background. 
The origin of the settlement is prior to the Roman colonization, with the precise function of a commercial site in the hilly hinterland, on an area comprising the Santerno River, the plain, the Senio River and the heights of the "Vena del Gesso".

The settlement had the physiognomy of the "aligned village": an elongated sequence of buildings overlooking the piedmont road. Although the genesis was probably free, the settlement was subsequently characterized by planning interventions to regulate its structure: first of all the birth of the Via Aemilia, which completely defined the route of the city's decumanus maximus, and constitutes the guide to transformation of the Imola area.

Through the observation of the dense road network that still develop in the city, the construction of a first regular quadrangular structure with sides of about $300 \mathrm{~m}$ was hypothesized; it was divided into four almost uniform regions by the decumanus and the cardo maximus.

The original settlement had been transformed into the small and orderly urban core of Forum Cornelii, as Imola was called. Despite the probable absence of urban walls, it constituted a territorial capital, a residential and commercial centre, with religious and political-administrative services.

In the following centuries, a rapid development took place, thanks to the economic, social and demographic progress of the city and the territory.

A rapid and total building renovation was carried out, resulting in a new rational urban planning that was completed in the Augustan age.

The limits of the city expanded, old roads were extended while others were traced, so that Forum Cornelii almost reached the extension of the medieval centre.

The city reached an almost rectangular area, over 300.000 square meters.

Forum Cornelii followed the typical pattern of cities of ancient foundation, based on two main roads, the decumanus maximus and the cardo maximus. The decumanus maximus was the Via Aemilia. The relative symmetry and orthogonality of the streets guaranteed an orderly appearance to the entire urban environment.

The streets organization was completed by other cardines and internal decumans dividing the town into rectangular aggregates, parallel to Emilia, which are often still recognizable in the form of the current ones. Starting from the II century AD, Imola went through a period of decline; historical sources testify its abandonment in the IV century.

Only after the XI century, after the acute phase of decline, the city changed.

The starting point of the new urban development coincided with a very narrow perimeter compared to the maximum extension reached by Forum Cornelii, but the new villages began to flourish and gradually led the city to occupy the area that it still covers today (figure 2).

After this first phase of analysis, the historical research was combined with the consultation of archival documents, reaching the most precise definition of the transformations undergone by the different building aggregates.

These documents referred to the post-unitary cadastral plans and estimated appraisals between the eighteenth and nineteenth centuries, where the buildings were also described through schematic graphical representations.

The survey activity allowed to understand the constructive characterization and the construction components of the building aggregates, which was then represented in specific drawings.

This phase provided the framework for carrying out the assessment phase.

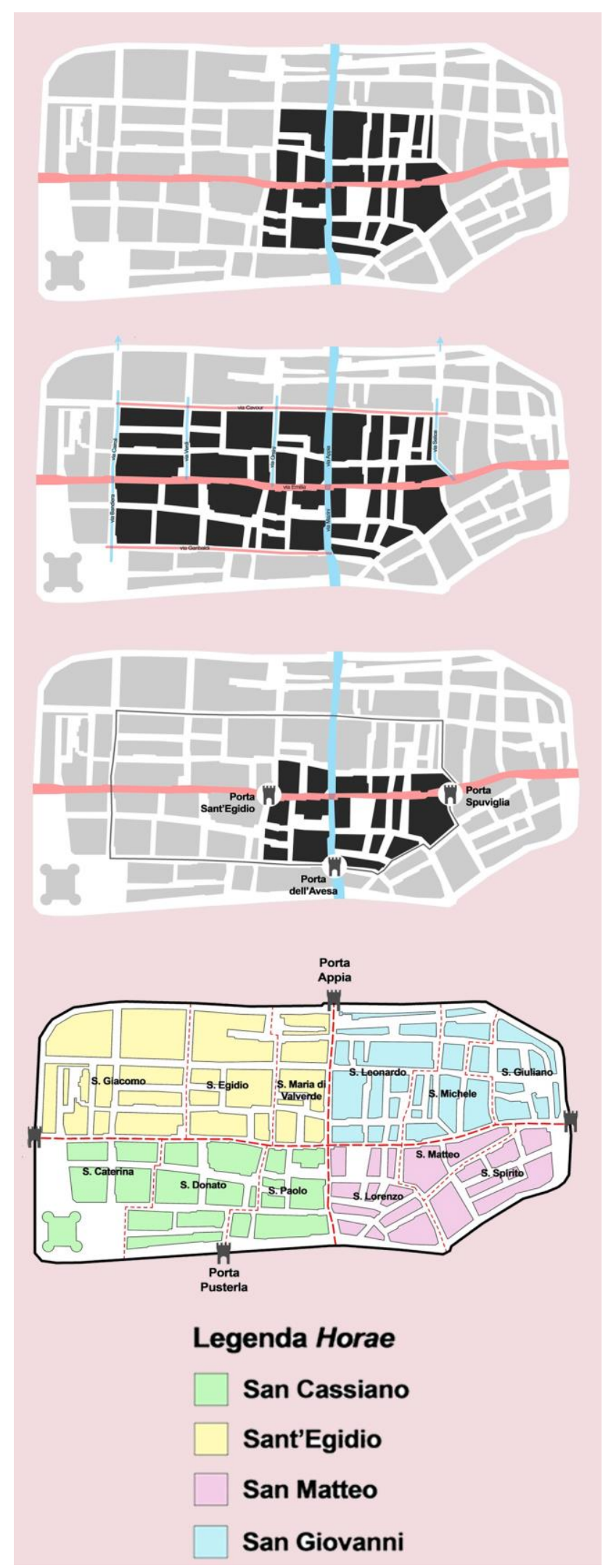

Figure 2. Evolution of the urban layout of Imola; from top to bottom: III century BC, I century BC, XI and XIV century AD (drawings by Laura Masi) 


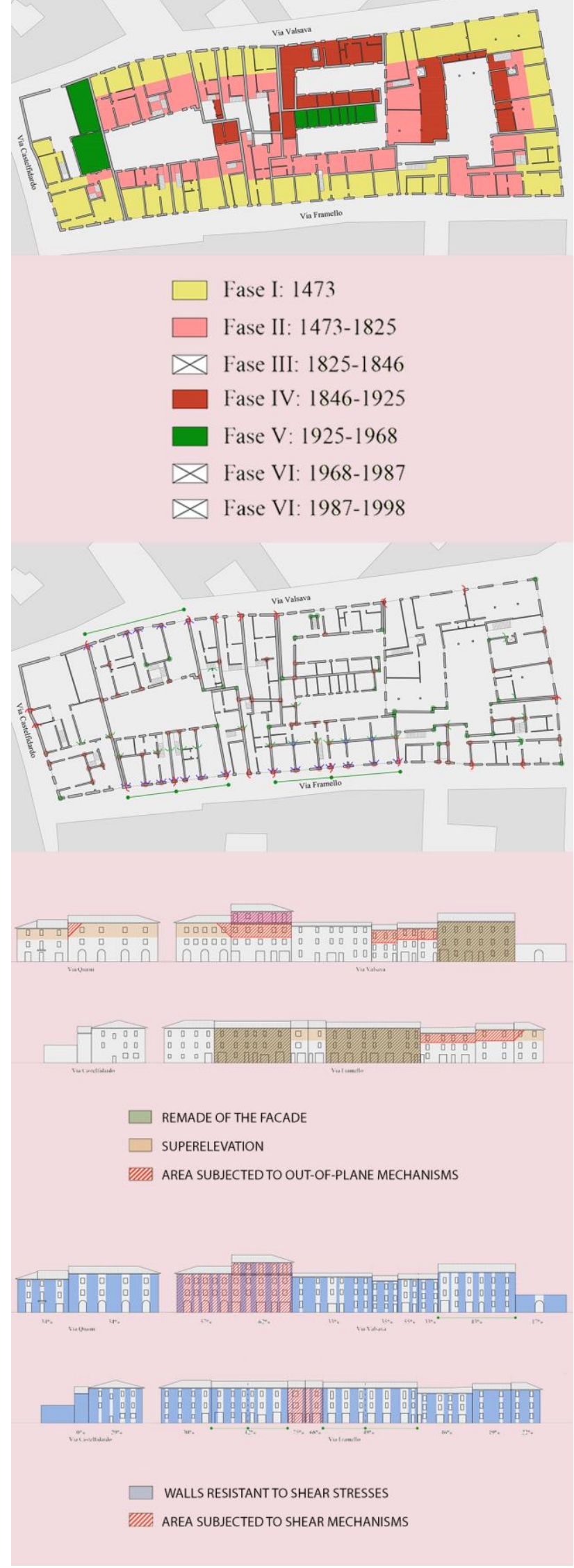

Figure 3. Indicators used for the seismic assessment Summary of the seismic vulnerability assessment for one of the analyzed building aggregates; from top to bottom: historical evolution, identification of wall disconnections, façade areas subjected to overturning, thrusts deriving from the covering layout, walls with weak shear strength (drawings by Matteo Lobina)

The conformation of the first urban layout of Imola was based on a hypothesis now generally shared; this consisted in the adoption of rectangular-shaped lots where the houses were built, with the smaller side along the main roads, coinciding with the long sides of the buildings.

This layout -defined as "gothic lot"- is confirmed by the historical iconography, which shows a subdivision of the centre into building aggregates with the described characteristics (Predari, Mochi, Gulli, 2014).

The buildings were concentrated along the streets and they left wide open spaces inside; over the centuries, these spaces have been affected by enlargements with a constant increase in building density.

This density was further increased with the raising of buildings, from the original two levels to the height of four floors. In addition to the transformation processes already mentioned, often these modifications were characterized by constructive solutions not designed to guarantee safety during seismic events.

For the transformations in the last two or three centuries, the archival research has allowed to record the processes of modification of the historical buildings with great reliability.

In the final phase of assessment, a damage scenario was obtained for each building aggregate, through the methodology based on the described indicators (figure 3 ).

The global analytical index referring to the entire building aggregate is obtained from the sum of all the partial vulnerability indicators, multiplied by corrective coefficients that derive from the danger and frequency of damage.

The highest coefficients are linked to overturning damage, while those related to shear stresses are lower.

The maximum value that the seismic vulnerability index can assume is $100 \%$; for the analyzed aggregates in Imola, the values vary from $20.21 \%$ to $37.84 \%$.

\section{THE SURVEY PROCEDURE}

Since ancient times, the metric survey of urban centres has used cartography as a means to transmit geometries to possible users, but nowadays one of the best techniques for surveying and monitoring urban centres is the Structure from Motion.

The developments of digital photogrammetry are oriented to the ability to automatically generate models of objects, which are always closer to reality, thanks to the progress of computer vision.

In particular, the Structure from Motion systems allow us to estimate the 3D position of points of different images, reconstructing the geometry of the object represented (structure) and the position of the camera (motion) (Aicardi, Chiabrando, Lingua, Noardo, 2018).

Structure from motion technique is a digital technology able to generate three-dimensional clouds of points departing from simple raster images.

It represents an efficient way to recollect useful information on the current state of existing buildings. Among its distinctive features are: precision, reliability, portability, rapidity of execution, operating simplicity and moderate costs. Metashape was employed for 3D photo-modeling.

Photo elaboration with Metashape ensured the identification of the object's salient points, which allowed to control the uncertainty level of metric values, always $<3 \mathrm{~mm}$.

In the majority of cases, we were able to obtain a complete reconstruction of the object overcoming major problems of 
coverage. We are talking about a kind of software operating through the automatic recognition of homogeneous points comparing the pictures, recognized through an artificial view of informatics algorithms (Wendel, Irschara, Bischof, 2011).

The program is able to align the pictures with prospective view's mechanisms, creating a Sparse Cloud of points. Once the whole photographic set is aligned and the error allocated, it thickens the points then shaping a Dense Cloud by applying stereophotogrammetry formulas.

Dense Cloud's points are then interpolated to produce the threedimensional surface called "mesh".

Elected pictures are automatically screened on the mesh and mixed with one another generating in this way the complete object's texture.

Metashape proceeds all along the elaboration according to these following generation phases of the model: photos alignment, cloud building, mesh building, model scaling, model and texture exporting.

It was essential to clean the cloud of points and the mesh after each calculating phase in order to erase the majority of disturbing points, being at the same time focused on keeping the useful ones. Further correcting operations on the mesh were carried out, like for example hole's closure interventions.

As the group of pictures to be elaborated had a great extension, it was first necessary to elaborate each single complex (one corresponding to only one chunk), then joining more chunks before creating the mesh.

Metashape automatically cleans the pictures from extraneous disturbing elements. It was anyway possible to erase such details by exporting the texture under jpeg format and adjusting it using an editing software.

Likewise, we can internally hide the disruptive component and consequently re-calculate the texture in a proper way. Finally, we proceeded to survey each spatial coordinate referring to all the points to be connected with the scaled model.

The continuous evolution of survey techniques and 3D modeling based on photogrammetry appears increasingly important and necessary for the interpretative phase, in the conservation and storage of data for the valorisation of the built environment, having a particularly rich value, such as the Emilian one (Recky, Wendel, Leberl, 2011).

The interest in different techniques and methods of digital survey and three-dimensional modeling in the technical field is highlighted by the growth in demand, their increasingly frequent use and the need to increasingly use forms of digital recording and archiving at different levels of scale and resolution.

Obviously, the use of these tools is part of a well-coded cognitive process, where particular attention must be paid precisely to the data acquisition and processing phase (Meixner, Leberl, Brédif, 2011). As regards the data acquisition, the mainly adopted photographic shooting technique was the parallel axis technique, ideal for architectural complexes that extend considerably in length. This technique consists of taking multiple photographs, moving parallel to the façade of the building and taking pictures with a frame that surpassed the previous box by at least $25 \%$. This method made it possible to draw up the façade design and was ideal for extracting the surface texture.

In this case, shooting was performed with the camera in a portrait position to take advantage of the maximum resolution of the camera.

Furthermore, another technique was used, the shooting with converging axes technique, although not in the best way because of the road size, which was too small and did not allow a perfect shooting: it consists in turning around the building and making a sort of semicircle and aiming in the barycentre of the façade.

It was not always possible to frame the entire façade for all shootings, as desirable, and it was sometimes necessary to take pictures to portions of the building. Normally, the photos were acquired by turning in a semi-circle with intervals of $30^{\circ}(5$ shots per semicircle).

The photo sets were made using the Canon EOS 600D camera. The environmental conditions, linked above all to the elevation of the buildings and to the presence of very narrow streets, involved the use of a lens $18-135 \mathrm{~mm}$.

The Canon EF-S 18-135mm lens is a standard to short telephoto telezoom for Canon digital single-lens reflex cameras with an EF-S lens mount.

The field of view has a $35 \mathrm{~mm}$ equivalent focal length of 29 $216 \mathrm{~mm}$. With its $7.5 \times$ zoom range, it is placed into the superzoom category.

Canon offers further lenses with even higher zoom ranges, such as the EF-S $18-200 \mathrm{~mm}$ lens $(11 \times$ zoom range). The software used for data processing is Agisoft Metashape (new realease of Agisoft Photoscan). Metashape is a stand-alone software product that performs photogrammetric processing of digital images and generates 3D spatial data to be used in GIS applications, cultural heritage documentation, and visual effects production as well as for indirect measurements of objects of various scales. The model produced was generated on a total number of photographs equal to 316 .

The photographs were loaded into the software by subdividing them into different chunks. Metashape consented to generate a polygonal model and texture in HD. Through feature extraction and matching operations, as the recognition of Tie Points which are common to more images, and their gathering thanks to Scale-Invariant Feature Trasform algorithm we achieved a three dimensional model composed by TiePoints, useful for the orientation (sparse point cloud) to whom the dense matching followed: during this phase the algorithm allowed to generate a points made model of the interested object called dense point cloud, once analysed orientated photograms with an established field partition. Then the scaling and referencing of the model was done by means of measurements or known points recollected on field, with mesh creation and projection of HD oriented pictures on the achieved mesh model.

In particular:

photos alignment: align the photographs with each other producing a cloud of points (sparse cloud);

building dense point cloud: once the whole set is aligned and the error is distributed, it produces a dense cloud building, through the classic stereophotogrammetry formulas;

building mesh: interpolating the points of the dense cloud produces a three-dimensional surface (mesh building);

- $\quad$ building model texture: on the mesh the photographs are projected mixing them one with the other, thus generating the complete texture (texture building)

The creation of a kind of model which is measurable and navigable is clearly a great advantage not only to document and monitorate buildings, but also to the evaluation procedure, intended to prevent and reduce the vulnerability (Letellier, Eppich, 2015) (figure 4, figure 5, figure 6).

In particular, from the three-dimensional model obtained by Metashape it is possible to extract orthophotoplans useful for the analysis of vulnerability, in fact orthophotos and photoplans allow a significant reduction of reading times and direct survey, for example of wall textures and degradation, as it is guaranteed the possibility of realizing measures and detailed analysis. 


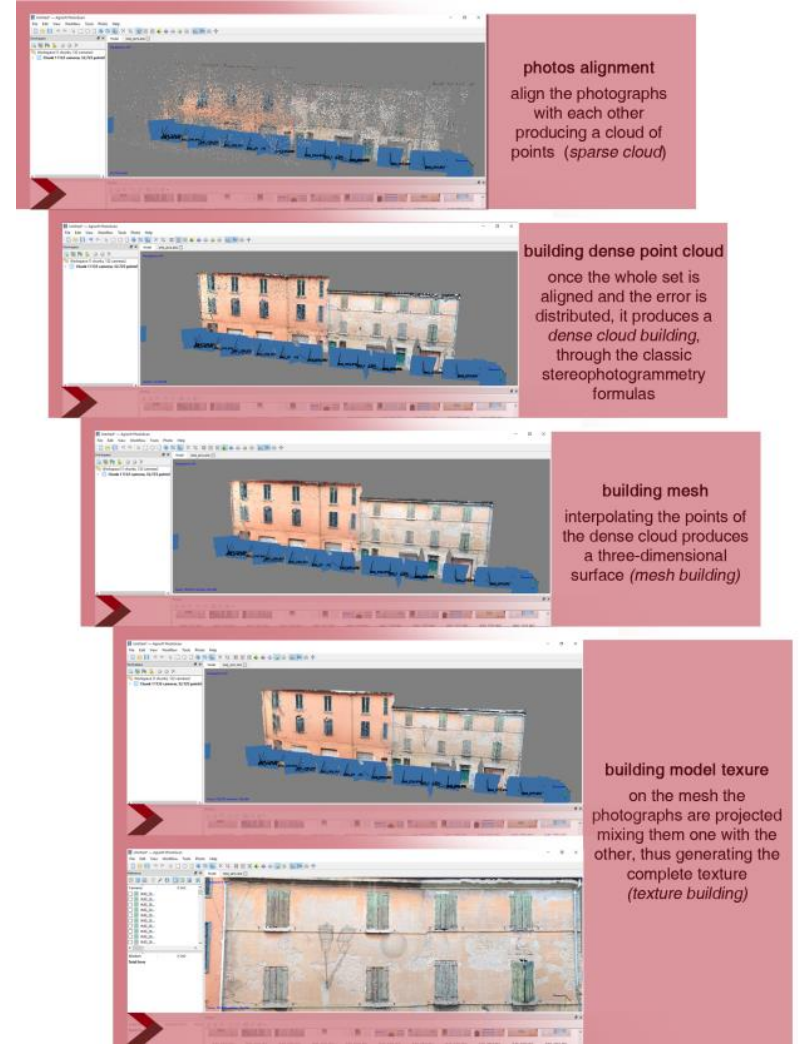

Figure 4. Model creation.

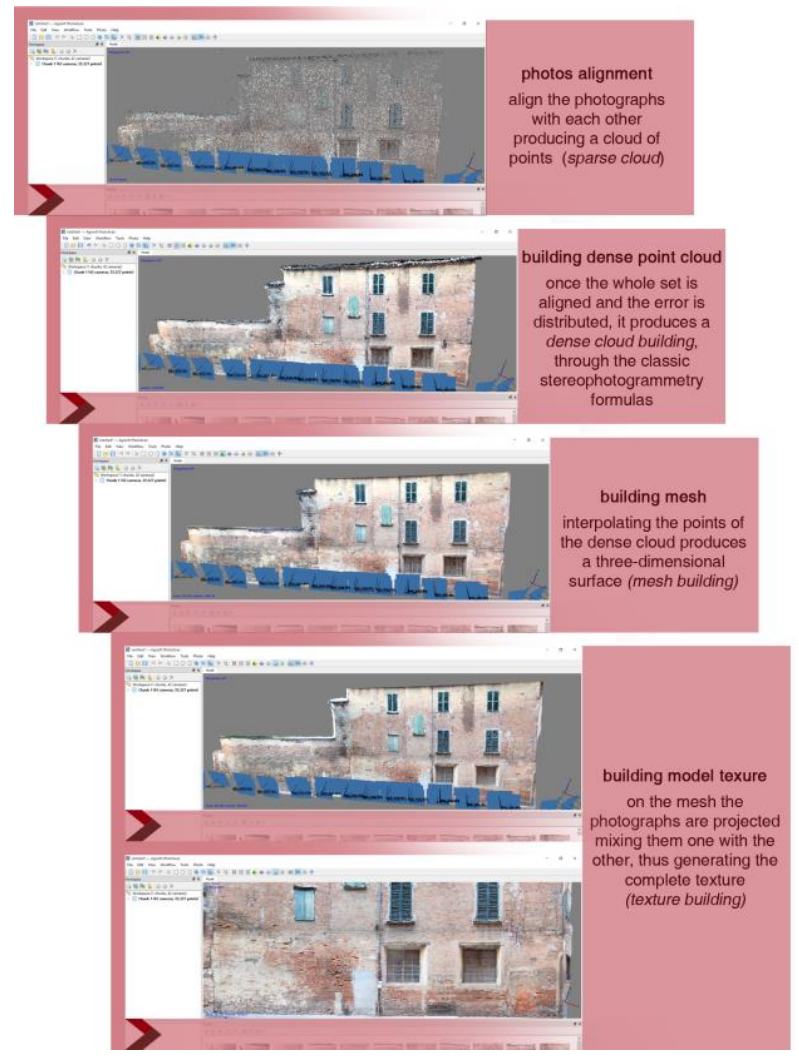

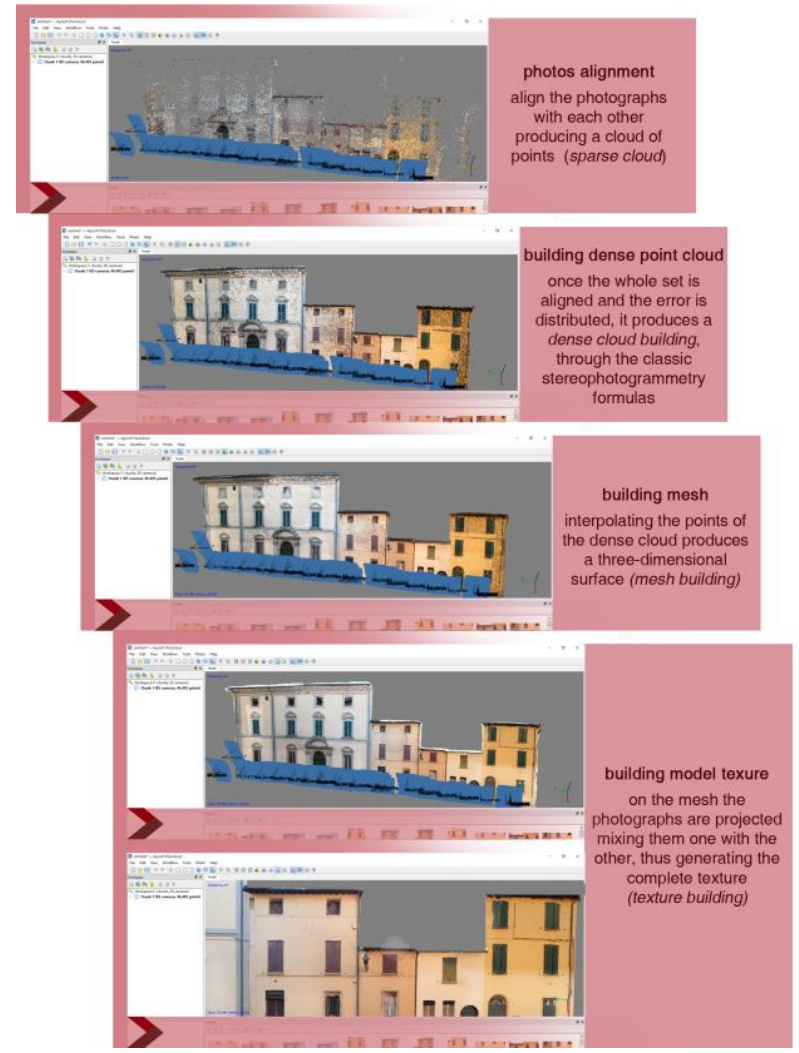

Figure 6. Model creation.

\section{CONCLUSIONS}

The appeal of urban surveys is to try and portray large parts of the anthropised city using iconic models. Models that also allow to interpret many buildings, aggregates and large anthropised areas. The survey operation, as a process involving extensive knowledge, aims at the accurate representation of the analysed subject; for this reason, drafting a procedure that uses the interaction between direct and indirect low cost methodologies and their elaboration for the realization of $2 \mathrm{D}$ and $3 \mathrm{D}$ models would be a topic of great interest, to make the final scientific results more objective and correct.

Metric survey methods record the position and size of the elements of an object, but also allow the visualization of the relationship between elements, highlighting information related to the consistency of the material and the state of preservation. The accuracy of the point cloud model of the historic centre of Imola can be verified in the scientific sense since the values of the measured dimensions do not deviate much from their true value.

Furthermore, the model provides an exhaustive description, though limited to the external walls of the buildings, which are the subject of investigation. All this is really useful in relationship with a quick evaluation of the seismic vulnerability since it constitutes the basis for a rapid but precise survey useful for the purposes of graphic restitution and the characterization of the fronts of the building aggregates before the seismic assessment.

Figure 5. Model creation. 


\section{REFERENCES}

Aicardi, I., Chiabrando, F., Lingua, A.M. Noardo, F., 2018. Recent trends in cultural heritage 3D survey: The photogrammetric computer vision approach. J. Cult. Heritage.

Azhar, S., 2011. Building Information Modeling (BIM): Trends, Benefits, Risks, and Challenges for the AEC Industry. In: Leadership and Management in Engineering, Vol. 11(3).

Barrile, V., Gelsomino, V. Bilotta, G., 2017. UAV and Computer Vision in 3D Modeling of Cultural Heritage in Southern Italy. IOP Conference Series: Materials Science and Engineering 225.

Beolchini, G.C., Milano, L., Antonacci, E., 2005. Repertorio dei meccanismi di danno, delle tecniche di intervento e dei relativi costi negli edifici in muratura. Definizione di modelli per l'analisi strutturale degli edifici in muratura, Volume II, Parte Ia. Convenzione di Ricerca con la Regione Marche; Consiglio Nazionale delle Ricerche - Istituto per la Tecnologia delle Costruzioni - Sede di L'Aquila; Dipartimento di Ingegneria delle strutture, delle Acque e del Terreno (DISAT) - Università degli Studi de L'Aquila.

Bianchini, C., Ippolito, A., Bartolomei, C., 2015. The surveying and representation process applied to architecture: non-contact methods for the documentation of cultural heritage. In: Brusaporci, S. (a cura di), Handbook of Research on Emerging Digital Tools for Architectural Surveying, Modeling, and Representation. Hershey: IGI Global.

Cangi, G., 2012. Manuale del recupero strutturale $e$ antisismico. Dei, Roma.

Carocci, C.F., 2005. La lettura critica del costruito dei centri storici. In: Rischio sismico, territorio e centri storici. Atti del Convegno Nazionale, Sanremo (IM) 2-3 luglio 2004, Lagomarsino S., Ugolini P. (ed). Franco Angeli, Milano.

Consiglio Superiore dei Lavori Pubblici, Gruppo di lavoro istituito con nota del Presidente del Consiglio Superiore dei Lavori Pubblici n. 7547 del 06/09/2010, Studio propedeutico all'elaborazione di strumenti d'indirizzo per l'applicazione della normativa sismica agli insediamenti storici, 20/04/2012.

Coppo, S., 2010. Contenuti e finalità del rilievo urbano. In: Coppo, D., Boido, C., (a cura di). Rilievo Urbano. Conoscenza e rappresentazione della città consolidata, Alinea, Firenze.

Cremonini, I., 1994, Rischio sismico e pianificazione dei centri storici (edited by), Alinea, Firenze.

Lagomarsino, S., Ugolini, P., 2005. Rischio sismico, territorio e centri storici. FrancoAngeli, Milano.

Letellier, R., Eppich, R., 2015. Recording, Documentation and Information Management for the Conservation of Heritage Places.

Meixner, P., Leberl, F., Brédif, M., 2011. Interpretation of 2D and 3D building details on facades and roofs. In: PIA 2011 Conference on Photogrammetric Image Analysis, Munich, 5-7 October 2011, Int. Arch. Photogramm. Rem. Sens. Spatial Inform. Sci. 38(3/W22), International Society for Photogrammetry and Remote Sensing (ISPRS), Munich, Germany, 137-142.
Ministero per i Beni e le Attività Culturali - Dipartimento per i Beni Culturali e Paesaggistici - Direzione Generale per i Beni Architettonici e Paesaggistici, 2006. Linee Guida per la valutazione e riduzione del rischio sismico del patrimonio culturale, Gangemi Editore, Roma, 5.

Mochi, G., 2009a. Processo tipologico e sicurezza sismica \#2. In: Il sisma. Ricordare, prevenire, progettare, AA.VV, Alinea, Firenze, 539-551.

Mochi, G., Predari, G., 2016b. La vulnerabilità sismica degli aggregati edilizi. Una proposta per il costruito storico. Edicom edizioni, Monfalcone.

Predari, G., Mochi, G., Gulli, R., 2014. The transformation process of masonry buildings in historic towns: the case of Medicina in northern Italy, "CONSTRUCTION HISTORY», $29,1-20$.

Recky, M., Wendel, A., Leberl, F., 2011. Façade segmentation in multi-view scenario. In: International Conf. on $3 D$ Imaging, Modeling, Processing, Visualization and Transmission [3DIMPVT 2011], IEEE Computer Society Proceedings, University of Hangzhou, Hangzhou, PR China, 358-365.

Wendel, A., Irschara, A., Bischof, H., 2011. Automatic alignment of $3 \mathrm{D}$ reconstructions using a digital surface model. In: Proc. IEEE International Conference on Computer Vision and Pattern Recognition (CVPR), Workshop on Aerial Video Processing, IEEE Computer Society, Washington, DC, 29-36. 